\title{
Cumulative Antenatal Risk and Kindergarten Readiness in Preterm-Born Preschoolers
}

\author{
Andrew M. Heitzer ${ }^{1} \cdot$ Jamie C. Piercy ${ }^{1} \cdot$ Brittany N. Peters $^{1}$ - Allyssa M. Mattes ${ }^{1} \cdot$ Judith M. Klarr $^{2} \cdot$ Beau Batton $^{3}$. \\ Noa Ofen ${ }^{1,4} \cdot$ Sarah $\operatorname{Raz}^{1,5}$ (D)
}

Published online: 16 August 2019

(C) Springer Science+Business Media, LLC, part of Springer Nature 2019

\begin{abstract}
A suboptimal intrauterine environment is thought to increase the probability of deviation from the typical neurodevelopmental trajectory, potentially contributing to the etiology of learning disorders. Yet the cumulative influence of individual antenatal risk factors on emergent learning skills has not been sufficiently examined. We sought to determine whether antenatal complications, in aggregate, are a source of variability in preschoolers' kindergarten readiness, and whether specific classes of antenatal risk play a prominent role. We recruited 160 preschoolers (85 girls; ages 3-4 years), born $\leq 33 \frac{6}{7}$ weeks' gestation, and reviewed their hospitalization records. Kindergarten readiness skills were assessed with standardized intellectual, oral-language, prewriting, and prenumeracy tasks. Cumulative antenatal risk was operationalized as the sum of complications identified out of nine common risks. These were also grouped into four classes in follow-up analyses: complications associated with intra-amniotic infection, placental insufficiency, endocrine dysfunction, and uteroplacental bleeding. Linear mixed model analyses, adjusting for sociodemographic and medical background characteristics (socioeconomic status, sex, gestational age, and sum of perinatal complications) revealed an inverse relationship between the sum of antenatal complications and performance in three domains: intelligence, language, and prenumeracy ( $p=0.003,0.002,0.005$, respectively). Each of the four classes of antenatal risk accounted for little variance, yet together they explained $10.5 \%, 9.8 \%$, and $8.4 \%$ of the variance in the cognitive, literacy, and numeracy readiness domains, respectively. We conclude that an increase in the co-occurrence of antenatal complications is moderately linked to poorer kindergarten readiness skills even after statistical adjustment for perinatal risk.
\end{abstract}

Keywords Prenatal $\cdot$ Prematurity $\cdot$ Preschool $\cdot$ Neuropsychology $\cdot$ School readiness

Electronic supplementary material The online version of this article (https://doi.org/10.1007/s10802-019-00577-8) contains supplementary material, which is available to authorized users.

\section{Sarah Raz}

sarahraz@wayne.edu

1 Department of Psychology, Wayne State University, Detroit, MI, USA

2 Department of Newborn Medicine, William Beaumont Hospital, Royal Oak, MI, USA

3 Department of Pediatrics, Southern Illinois University School of Medicine, Carbondale, IL, USA

4 The Institute of Gerontology, Wayne State University, Detroit, MI, USA

5 Developmental Neuropsychology Laboratory, the Merrill-Palmer Skillman Institute, Wayne State University, 71 East Ferry, Detroit, MI 48202, USA
A substantial body of literature is dedicated to scholastic achievement of children born preterm, with varying degrees of prematurity, in comparison to their term-born peers. Quantitative integration of findings from elementary, middle, and high-school children born $\leq 33$ weeks gestation yields effect sizes within the medium to medium-large range for the three core domains of academic performance (reading, spelling, and mathematics), as measured by standardized achievement tests (Aarnoudse-Moens et al. 2011). These results are consistent with findings from quantitative integration of cognitive outcome studies of children born preterm (KerrWilson et al. 2012).

Deficiencies in academic skills of school-age children born preterm are heralded by the emergence of domain-specific, neuropsychologically-based, preliteracy and prenumeracy skill deficits during the preschool years. Indeed, comparisons of performance on academic readiness tasks between preterm- 
born preschoolers and their term-born peers yielded poorer scores in the former group, regardless of gestational age. Group differences have been documented in expressive and receptive language abilities as well as in visuomotor or graphomotor (preprinting) skills in both very preterm (Foster-Cohen et al. 2010; Caravale et al. 2005; Torrioli et al. 2000) and late-preterm (Baron et al. 2009; Baron et al. 2011) cohorts.

Establishing the nature of early biomedical risk factors that forecast deficits in critical academic precursor skills is essential for identification of preschoolers at risk for deviation from the typical neurodevelopmental trajectory. Findings from a recent quantitative integration of the literature suggest that both preterm birth and adverse antenatal factors are important antecedents of intellectual disability diagnosed between 1 and 17 years of age (Huang et al. 2016). Yet few preschool outcome studies included within-group examination of the links between complications associated with preterm-birth and performance on neuropsychological tasks that tap domain-specific, literacy or numeracy, precursor skills. Foster-Cohen et al. (2010), focusing on the impact of peri-, and neonatal, but not antenatal, complications, reported no significant associations between several early risk factors (including the sum of perinatal complications) and language delay within a cohort of four-year-old preschoolers born very preterm. In a similar sample of preschoolers born $<34$ weeks gestation Torrioli et al. (2000) were able to document a significant relationship between a single major antenatal complication, intrauterine growth restriction, and intelligence (but not visual-motor integration).

Consistent with the prevailing fetal programming framework (Barker 1998), conditions or risks originating in-utero have the unique capacity to modify long-term physical health and behavioral outcome. Though the exact mechanisms leading to disease or cognitive-behavioral deficits have yet to be specified, it has been suggested that fetal adaptation to environmental stress may involve vascular, metabolic or endocrine changes that permanently alter bodily structure or function (Hocher et al. 2001). Antenatal perturbations are likely transmitted to the infant through their effects on placental function. The latter, in turn, adversely influences fetal and postnatal brain development and cognitive-behavioral outcome (Zeltser and Leibel 2011; Buss et al. 2012; Davis and Sandman 2012).

Within the high-risk group of preterm-born children, both the variability in the base-rates of various antenatal complications associated with prematurity and the sheer number of medical risk factors that require consideration often impede exploration of developmental outcome effects of early biological adversity. Additionally, the developmental impact of discrete medical complications is probably cumulative (Shalev et al. 2014). Cumulative risk indices may show increased stability across developmental periods, accounting for more outcome variability between individuals than specific complications (Wade et al. 2015). Aggregate scores that reflect cumulative risk associated with a distinct early risk epoch may be increasingly sensitive compared to discrete complications that are likely linked to small effects that are difficult to detect (Whitehouse et al. 2014). Although the influence of cumulative perinatal risk on developmental outcome of preterm children has received some consideration (e.g., Carmody et al. 2006; Foster-Cohen et al. 2010), the effects of cumulative antenatal risk in this vulnerable group remain essentially unexplored. Hence, our chief objective was to examine the combined contribution of common antenatal complications to explaining individual differences in academic (kindergarten) readiness within a preterm-born cohort. In addition to gauging intellectual abilities, we evaluated language skills as precursors of reading attainment, visual-motor integration skills as antecedents of writing-skill development, and early number concepts as the preschool forerunners of math achievement. We predicted that degree of antenatal risk in preterm-born preschoolers will be inversely related to both general cognitive, and domain-specific, neuropsychological skills that provide the basis for scholastic achievement.

\section{Method}

\section{Participants}

Preterm-born children ( $<34$ weeks' gestation) were recruited for the study between 3 and 4 years of age and evaluated between May 2011-July 2016. The children were born at William Beaumont Hospital (WBH), Royal Oak, MI, in 2007-2012, and treated in the Neonatal Intensive Care Unit (NICU). At WBH-NICU resuscitation is attempted for all infants with an estimated gestational age $\geq 23 \%$ weeks (Batton et al. 2011). Children with congenital anomalies, or who required mechanical ventilation after discharge, were excluded from the retrospectively identified NICU cohort matching our inclusion criteria. The families of $41.63 \%$ of 860 cases could still be reached for recruitment attempt based on contact information provided at the time of birth (see Online Resource 1). Of these, families of $38.27 \%$ cases were not interested, for multiple reasons. In accord with WBH Human Investigation Committee guidelines nonparticipating families were not specifically queried, yet amongst common reasons spontaneously provided by families for nonparticipation were being "too busy", residing too far away or not wanting to travel from suburban areas to the city. Families of 51 additional cases (14.24\% of those successfully contacted) "no-showed" for the schedule assessment twice and were not rescheduled. The 170 available participants constituted $51.20 \%$ of the pool of cases whose families could be contacted between 3 and 4 years of age. 
Of 170 evaluated cases ten were excluded from data analyses, five with suspected antenatal substance exposure and five with missing background medical information pertinent to this investigation. Altogether 160 cases were available for this study (see Table 1 for sociodemographic characteristics). As the table shows, our sample included participants from a very broad socioeconomic range. Nonetheless, the relatively high mean SES (48.6 of 66 possible points; SD = 10.00) reflects the composition of the catchment area of WBH. This region includes primarily middle social strata, thereby minimizing the possibility of confounding the effects of antenatal risk with other adverse environmental factors associated with socioeconomic status. Correspondingly, about $85 \%$ of NICU admissions were covered by private medical insurance and only $15 \%$ by Medicaid. Additionally, $78.1 \%$ of the mothers and $62.3 \%$ of the fathers attained a college degree. The final sample included $75(46.9 \%)$ boys and 28 (17.5\%) participants of African-American descent.

The proportion of males to females in our sample (46.9\%: $53.1 \%$ ) was not significantly different from the proportion observed in the remaining portion of the total relevant NICU cohort from which we attempted to recruit (53.14\%: $\left.46.86 \% ; \chi^{2}[1]=2.05, p=0.15\right)$. The proportion of singletons to multiples in our sample (56.3\%: $43.8 \%)$ was also not significantly different from the proportion observed in the remainder of the total cohort $\left(60.71 \%: 39.29 \% ; \chi^{2}[1]=1.08\right.$, $p=0.30)$. Information about racial distribution was available for two thirds of the total relevant cohort. As noted above, our

Table 1 Demographic and socio-familial characteristics for total sample

\begin{tabular}{ll}
\hline Characteristic & Mean (SD) /Frequency (\%) \\
\hline Adjusted age (months) $^{\mathrm{a}}$ & $44.8(3.1)[38.6-53.1]$ \\
Multiples (S:M) $^{\mathrm{b}}$ & $90: 70(56.3: 43.8)$ \\
${\text { Race }(\mathrm{W}: \mathrm{O})^{\mathrm{c}}}_{\mathrm{SES}^{\mathrm{d}}}$ & $126: 34(78.8: 21.2)$ \\
Sex (M:F) & $48.6(10.0)[24.0-66.0]$ \\
Parental VIQ $^{\mathrm{e}}$ & $75: 85(46.9: 53.1)$ \\
Mother's education (yrs.) & $101.7(11.6)\{135\}[70.0-136.0]$ \\
Father's education (yrs.) & $16.2(1.8)[11-20]$ \\
Bilingual home & $15.4(2.1)[10-20]$ \\
Preschool attendance & $12\{155\}(7.7)$ \\
\hline
\end{tabular}

Frequencies (with percentages in parentheses) are reported for discrete data, means (with standard deviations in parentheses) for continuous data. For missing data number of cases provided in braces. Ranges in brackets

${ }^{\mathrm{a}}$ Age adjusted for prematurity

${ }^{\mathrm{b}} \mathrm{S}=$ single, $\mathrm{M}=$ multiple (61 twins and 9 triplets)

${ }^{\mathrm{c}} \mathrm{W}=$ White; $\mathrm{O}=$ Other (one or both parents not Caucasian)

${ }^{d}$ Hollingshead's (1975) Four-Factor Index of Social Status.

e Prorated IQ based on three subtests (Vocabulary, Similarities, and Information) of the Wechsler Adult Intelligence Scale-IV; Testing administered to the biological mothers sample included $17.5 \%$ African Americans, a somewhat smaller proportion than that observed in the remainder of the relevant NICU cohort $\left(24.16 \% ; \chi^{2}[1]=3.01, p=.08\right)$. The mean gestational age $(30.4 \pm 2.6$ weeks $)$ and birth weight $(1,424 \pm 457 \mathrm{~g})$ in our sample approximated the mean gestational age $(29.8 \pm 2.8$ weeks $)$ and birth weight $(1,434.3 \pm$ $513 \mathrm{~g})$ available for the total relevant cohort. Likewise, the length of stay was also similar for our sample (43.42 \pm 34.17 days) and the total cohort (42.9 \pm 34.5 days), and the proportion of children requiring ventilation in our sample was not statistically different from that observed in the remaining portion of the total cohort $\left(36.5 \%\right.$ vs. $38.57 \% ; \chi^{2}[1]=0.29$, $p=0.585)$.

According to parental report, none of the children had sustained a severe head injury with loss of consciousness. Postnatal seizure history was reported for 11 cases, yet only three required anticonvulsant medications. Whereas the intellectual abilities of two of these three children fell well within the Average range, the remaining case manifested severe intellectual deficits. As Table 1 shows, ninety participants were singletons and seventy were products of multiple pregnancy. Sixty-four multiples were co-members of twinships or triplets and therefore shared antenatal risk. Descriptive statistics regarding pregnancy and perinatal risk in our sample were based on data obtained retrospectively from hospital records (Table 2). Additional information regarding intervention procedures is provided in Online Resource 2.

Gestational age in our sample ranged from $23 \frac{4}{7}-33^{6} / 7$ weeks and was determined by maternal dates and confirmed by early prenatal ultrasound in $>95 \%$ of cases. Three children with CP diagnosis (spastic diplegia) were included in the sample. Additionally, there were three cases with perinatal diagnoses of Grade III intraventricular hemorrhage, one with Grade IV, one with periventricular leukomalacia (PVL), one with both PVL and subsequent cerebral palsy (spastic diplegia), and one with Grade IV hemorrhage coupled with hydrocephalus (requiring reservoir placement). Adding the aforementioned single seizure case with severe intellectual impairment, the "significant brain injury" subgroup included 11 cases. Importantly, statistical analyses were performed both with and without these cases.

\section{Evaluation of Pre-Kindergarten Readiness Skills}

General Assessment Considerations Evaluations were conducted between 2011 and 2016 by clinical psychology graduate students who had been extensively trained in developmental neuropsychological assessment. They were kept uninformed about participants' medical background data, with the single exception of being aware that the children were NICU graduates. All testing and other data collection procedures were conducted in compliance with ethical standards of the Helsinki 1964 Declaration and its later amendments, as well 
Table 2 Antenatal and perinatal background for the total sample of 160 cases

Antenatal

Perinatal

Pregnancy - general

Mother's age at delivery (years)

Parity

Smoking ( $\geq 1$ cigarette per day)

List of antenatal complications with frequency $>5 \%$

Abruption or suspected abruption of the placenta ${ }^{\mathrm{a}}$

Chorioamnionitis $^{\mathrm{b}}$

Diabetes $^{\mathrm{c}}$

HELLP syndrome ${ }^{\mathrm{d}}$

Hypertensive disorder in pregnancy ${ }^{\mathrm{e}}$

Hypothyroid $^{\mathrm{f}}$ (requiring hormone replacement)

Membranes ruptured $>12$ hours $^{\mathrm{g}}$

Placenta previa

Small for gestational age $(<10 \text { th centile })^{\mathrm{h}}$

Mean sum of antenatal complications ${ }^{i}$

$32.7(4.5)[159]$
$0.60(0.9)[158]$
$7(4.4)[159]$
$20(12.5)$
$33(20.6)$
$20(12.5)$
$11(6.9)$
$56(35.0)$
$30(18.8)$
$34(21.3)$
$16(10.0)$
$19(11.9)$
$1.50(1.00)$

Birth and delivery

Abnormal presentation ${ }^{\mathrm{j}}$

score at $5 \mathrm{~min}<6$

$5(3.1)$

Birthweight (grams)

Cord $\mathrm{pH}<7.1^{\mathrm{k}}$

Gestational age (weeks) ${ }^{1}$

$1,424(457)$

$5(3.2)[155]$

List of perinatal complications with frequency $>5 \%$

Anemia at birth ${ }^{\mathrm{m}}$

Bacterial infection $^{\mathrm{n}}$

Bronchopulmonary dysplasia $^{\circ}$

Hyaline membrane disease ${ }^{p}$

Hyperbilirubinemia $^{\mathrm{q}}$

Hypoglycemia $^{r}$

Intracranial pathology

Oxygenation required after discharge ${ }^{t}$

Patent ductus arteriosus ${ }^{\mathrm{u}}$

Mean sum of perinatal complications ${ }^{v, w}$
$21(13.1)$

$12(11.9)$

$27(16.9)$

$99(61.9)$

$17(10.6)$

$22(13.8)$

$27(16.9)$

$20(12.5)$

$38(23.8)$

$1.80(1.60)$

Frequencies (with percentages in parentheses) reported for discrete, means (with standard deviations in parentheses) for continuous, data. In case of missing data, $N$ provided in brackets

${ }^{a}$ Determined at delivery or by placental pathology

${ }^{\mathrm{b}}$ Includes only cases confirmed via histopathology (with or without funisitis)

${ }^{\mathrm{c}}$ Includes both gestational diabetes and diabetes mellitus

${ }^{\mathrm{d}}$ Hemolysis, Elevated Liver enzymes, and Low Platelet count

${ }^{\mathrm{e}}$ Including diagnoses of preeclampsia, chronic hypertension, and pregnancy induced hypertension

${ }^{\mathrm{f}}$ All cases requiring treatment with Levothyroxine

${ }^{\mathrm{g}}$ Time from preterm premature rupture of membranes to delivery. For the 34 cases membranes were ruptured from sixteen hours to 59 days (Mean \pm $\mathrm{SD}=240 \pm 306 \mathrm{~h}$ )

${ }^{\mathrm{h}}$ According to sex-specific, birthweight-for-gestational-age, reference norms (birthweight SD) published by Kramer et al. (2001)

${ }^{\mathrm{i}}$ Summary score for the nine above listed antenatal complications with sample frequency $>5 \%$

${ }^{\mathrm{j}}$ Includes any atypical presentations (breech, transverse lie, footling, etc.)

${ }^{\mathrm{k}}$ Arterial cord $\mathrm{pH}$ examined for 130 participants, arterial $\mathrm{pH}$ below 7.1 was recorded $(n=3)$. When only venous cord $\mathrm{pH}$ was available $(n=21)$, venous $\mathrm{pH}$ below 7.2 was recorded $(n=1)$. For four cases, initial capillary blood below 7.2 was recorded $(\mathrm{n}=1)$, whereas acid-base information was unavailable for five cases

${ }^{1}$ As determined by obstetrician; $>95 \%$ of cases were corroborated by antenatal ultrasound. Distribution: 33 cases $\leq 28 \% / 7$ weeks $(20.62 \%)+31$ cases $\leq 30$

$0 / 7$ weeks $(19.37 \%)+62$ cases $<326 / 7$ weeks $(38.75 \%),+34$ cases $\leq 336 / 7$ weeks $(21.25 \%)$

${ }^{\mathrm{m}}$ Initial hematocrit $<40 \%$

${ }^{\mathrm{n}}$ Established by positive blood culture

${ }^{\circ}$ Chronic lung disease: supplemental oxygen required at 36 weeks gestation or discharge for infants $<32$ weeks gestation. No cases were observed in this sample with gestational age $\geq 32$ weeks

${ }^{\mathrm{p}}$ Based on a chest roentgenogram and clinical evaluation

${ }^{\mathrm{q}}$ Peak bilirubin $\geq 12 \mathrm{mg} / \mathrm{dl}$

${ }^{\mathrm{r}}$ Diagnosed at least once during NICU stay

${ }^{\mathrm{s}}$ Documented on the basis of cranial ultrasound. (Mild = Bleed Grade 1\&2; Severe = Grade $3 \& 4$ using grading criteria by Volpe, 2001). Routine cranial ultrasound given to all infants with gestational age $\leq 32$ weeks, and when clinically indicated to infants with gestational age $>32$ weeks. There were twenty cases (12.5\%) with mild brain bleeds (sixteen Grade I and four Grade II) and seven (4.4\%) with severe intracranial pathology, including three Grade III intraventricular hemorrhage, two Grade IV (one requiring shunt), and two cases with periventricular leukomalacia

${ }^{\mathrm{t}}$ Infants discharged on the ventilator were not included in the current study

${ }^{\mathrm{u}}$ Diagnosed by clinical manifestations and echocardiographic information

${ }^{\mathrm{v}}$ Summary score for the nine above listed perinatal complications with sample frequency $>5 \%$

${ }^{\mathrm{w}}$ The rate of severe retinopathy of prematurity (> Stage 2) in the total sample was $4.11 \%$, below our inclusion cutoff (one cases with Stage III, four cases with Stage III+, and two cases with Stage IV) and there were no cases with Grade IV ROP after exclusion of eleven cases with significant neurological background. All cases with Stage III+ and Stage IV had received laser treatment

as with the regulations of the Human Investigation Committee of WBH and the Institutional Review Board of Wayne State
University. Informed consent was obtained from a parent/legal guardian of all individual participants included in the study. 
Cognitive Abilities Global intellectual functioning was evaluated using the Information (IN) and Block Design (BD) subtests from the Wechsler Preschool and Primary Scale of Intelligence (WPPSI-III; Wechsler 2002) in 96 cases, whereas 64 participants $(40 \%)$ were given the same subtests from the Fourth Edition (WPPSI-IV; Wechsler et al. 2012). For our participants' age, IN has excellent $\left(r_{\mathrm{xx}}=0.92\right)$, whereas BD shows good $\left(r_{x x}=0.85\right)$, internal consistency reliability (Wechsler 2002).

Prereading Skills Language skills were assessed using the Core Language (CL) score from the Clinical Evaluation of Language Fundamentals-Preschool (CELF-P2; Wiig et al. 2004). The CL stability coefficient for ages 3:0-3:11 is high $\left(r_{12}=0.87\right)$, whereas internal consistency and split-half reliability are excellent $\left(r_{x x}=0.91\right.$ and 0.92 , respectively). The CL scale is comprised of three subtests: Sentence Structure, Word Structure, and Expressive Vocabulary.

Prenumeracy Skills Quantitative knowledge and reasoning were measured using the Applied Problems (AP) subtest from the Woodcock Johnson (WJ-III) Tests of Achievement (Woodcock et al. 2001). At the prekindergarten level, AP assesses emergent counting, addition, and subtraction skills. The split-half reliability for ages 3 and 4 is excellent $\left(r_{11}=0.92\right.$ and 0.94 , respectively), and test-retest correlations range from $r_{12}=$ 0.85-.90 for ages 2-7 (Woodcock et al. 2001). Quantitative Concepts (QC), another WJ-III task that may be administered at three years of age does not possess an adequate floor (Alfonso and Flanagan 2002), and was therefore not included.

Preprinting Skills Eye-hand coordination skills were assessed using the Visual-Motor Integration (VMI) subtest from the Peabody Developmental Motor Scales (PDMS-2; Folio and Fewell 2000). The VMI includes items that require reaching and grasping, building with blocks, or copying designs. Internal consistency reliability for 36-47 months of age is excellent $\left(r_{11}=0.94\right)$, whereas the overall test-retest $\left(r_{12}=\right.$ $0.92)$ and inter-scorer $\left(r_{11}=0.98\right)$ reliability coefficients are also exceptionally high (Folio and Fewell 2000).

Descriptive Information for Performance Measures The mean FSIQ, CL, and VMI scores $( \pm \mathrm{SD})$ of our participants fell well within the Average range on three of the four readiness measures $(107.83 \pm 17.62,106.43 \pm 13.60$, and $10.73 \pm 2.91$, respectively), whereas mean AP score fell Above Average $(116.71 \pm 13.55)$. These favorable results are likely attributable to the preponderantly middle-class background of our sample. Notably, participants' scores spanned a broad range (with similar ranges and SD's for AP and CL) and it was our goal to explore the contribution of antenatal risk to explaining this variability. All standard scores were corrected for prematurity. The recalculation of the preterm preschooler's age-at- testing as time elapsed since the expected date of delivery allows one to derive standard scores based on age-reference norms of typical children who are similar in biological maturity. Additional descriptive data for the total-, and subsample without significant brain injury may be found in Online Resource 3.

\section{Measurement of Cumulative Antenatal and Cumulative Perinatal Risk}

Cumulative Antenatal Risk Consistent with the fetal programming hypothesis, our chief variable of interest was cumulative antenatal risk, operationalized as an index comprised of equally weighted complications. We included nine major complications with sample frequency $>5 \%$ (see Table 2) and with well documented relationships to unfavorable neonatal outcome or neurobehavioral sequelae. Specifically: hypertensive disorders (Maher et al. 2017), IUGR (Morsing et al. 2011), hemolysis, elevated Liver enzymes, and low platelet count [HELLP] syndrome (Barnhart 2015), hypothyroidism (Andersen et al. 2018, and Liu et al. 2018), diabetes (Fraser et al. 2012), chorioamnionitis (Yellowhair et al. 2018), preterm premature rupture of the membranes (PPROM; Pinto et al. 2018), placental abruption (Downes et al. 2017) and placenta previa (Weiner et al. 2016). Hence, for each participant we summed the identified complications, out of the nine relatively common antenatal risks, with the following sample distribution: 0 (15\%), 1 (40.62\%), $2(27.50 \%), 3(13.75 \%)$ and $4(3.10 \%)$. No cases were observed with frequency $\geq 5$ complications. The correlations between pairs of antenatal complications were moderate at best, suggesting that the information provided by discrete complications was not redundant. The strongest relationship was observed between diagnosis of histological chorioamnionitis and PPROM latency duration (time between membrane rupture and birth $)>12 \mathrm{~h}(r=0.489 ; p<0.001)$.

Cumulative Perinatal Risk A cumulative perinatal risk score was also computed and was included as a covariate. Nine major perinatal complications with sample frequency $>5 \%$ provided the basis for the perinatal summary score (see Table 2). Abnormal presentation was not included because this complication is thought to exert little influence on longterm outcome (Eide et al. 2005) and since the potentially unfavorable outcome effects are believed to be the result of confounding with the effects of prematurity or gestational age (Ismail et al. 1999). The latter variable was taken into account as a separate risk factor in this study.

Inter-Rater Reliability of Cumulative Medical Risk Indices Background medical information was obtained retrospectively from the mother's labor and delivery hospitalization as well as the NICU records. Intra-class correlations (ICC-2; Shrout 
and Fleiss 1979) for the antenatal and the perinatal complications composite scores were excellent, owing in part to the ease and efficiency of searching electronic records. Based on two independent and trained medical records reviewers, ICC equaled or approached unity for ten cases (ICC [9] $=1.00$, and 0.98 , for antenatal and perinatal composites, respectively).

\section{Construct Validity of Cumulative Medical Risk Indices}

Construct validity of the two summary scores was established in the singletons subsample $(N=90)$ by examination of the associations between the cumulative antenatal risk score and birth weight, an index of fetal growth and well-being, and between the cumulative perinatal risk score and both length of NICU stay and days on the ventilator, two indexes of need for medical intervention. Indeed, cumulative antenatal risk, adjusted for sex and gestational age, accounted for a significant portion of the variance in birth weight $(F[1,86]=5.024$, $p<0.028)$, whereas cumulative perinatal risk, adjusted for sex and gestational age, accounted for length of hospital stay $(F$ $[1,86]=56.11, p=0.028)$ and need for ventilation $(F[1$, $85]=36.47, p<.001)$.

\section{General Statistical Considerations}

In addition to the 90 singletons and seven single-members of twin pairs, our sample included 27 sets of co-twins and three sets of co-triplets. To capture the correlation between participants within sets of multiples, we used SPSS 24.0 MIXED (maximum likelihood) to fit separate linear mixed effects models for each outcome variable, with multiplicity (cases from the same birth mother) as a random effect. Thus, the individual multiples nested within each set were conceptualized as replications and were given a random block number that was unique, but identical for set members. In contrast, singleton children or multiples without an evaluated co-multiple had no replicates and were considered random block with size 1 . This model enabled both co-multiples and singletons to be used in the same analysis without either violating independence assumptions or, alternatively, discarding important information by including only a single member of each set.

The variable of interest, antenatal risk (the sum of antenatal complications), was entered together with sociodemographic covariates, sex and SES (Hollingshead 1975), into the model. The Hollingshead Index is a composite score reflecting family social status. The score is based on four factors: parental marital status, employment status, educational attainment and occupational prestige. Two additional covariates were entered to capture the influence of perinatal risk: gestational age and the perinatal complications summary score (see Table 2). Birth weight was highly correlated with gestational age and was therefore not included $(\mathrm{r}=0.82 ; p<0.001)$. Hence, the effect of antenatal risk on readiness was statistically adjusted for influence of four covariates, with all five predictors entered simultaneously into the model. Information about bivariate correlations among the five predictors may be found in Online Resource 4. One should note here that a fifth covariate, IQ test edition, was used in analyses of cognitive outcome.

The predicted variables were the cognitive (prorated FSIQ) and pre-academic (CELF-P2 CL score, WJ-III AP subtest score, and PDMS-2 VMI score) measures of kindergarten readiness corrected for degree of prematurity. Information about the correlations between the five predictors and four outcome measures is presented in Online Resource 5.

Three (1.9\%) of the 160 participants were unable to complete any of the tasks included in this evaluation (two cases with $\mathrm{CP}$ and a single case without significant neurological findings who required $>4.5$ months on the respirator at birth). Of the 148 children without significant brain injury who completed at least one task, a minority of cases failed to complete the tasks needed to obtain a score on one or more of the four preacademic performance indices $(n=1[0.6 \%], 8$ [5.4\%], 14 [9.4\%], and 6 [4.0\%] cases for FSIQ, CL, AP, and VMI, respectively). Due to the children's young age, it was difficult, if not impossible, to ascertain the causes of failure to attempt or follow instructions for specific subtests. Examination of the correlates of the sum of incomplete subtests per participant revealed significant associations neither with sociodemographic variables $(r[158]=-.102$ and -.100 for SES and sex; $p=.199$ and .207 , respectively) nor with preschool attendance $(r[155]=0.084 ; p=0.296)$. In contrast, a significant inverse relationship between the number of incomplete subtests and the FSIQ was observed $(r[156]=-.48$, $p<0.001)$. To avoid bias resulting from potential influence of the missing subtest scores, we applied multiple imputation to replace these performance data.

The results of data analyses are reported both before and after exclusion of cases with significant brain injury. Prior to data analyses, interactions between sex and the remaining predictors were examined for all outcome measures. As none of the interactions were significant (all $p$ values $>.15$ ), the reduced models were used. Follow-up analyses were conducted to explore whether any observed associations between antenatal risk and kindergarten readiness were attributable to a particular class of complications. Hence we grouped the nine antenatal complications comprising the summary score (Table 2) into four categories. These four groupings were based on shared etiological pathways, or shared antepartum symptoms coupled with at least some shared antecedents. Specifically, complications associated with either (1). intra-amniotic infection and inflammation (histologic chorioamnionitis and membranes ruptured $>12 \mathrm{~h}$; e.g., Stock et al. 2015); (2). placental insufficiency (hypertension in pregnancy, HELLP syndrome, and IUGR; e.g., Stepan et al. 2005); (3). maternal endocrine dysfunction (hypothyroid and diabetes; e.g., Haddow et al. 2016) or (4). uteroplacental bleeding (abruption and previa; e.g., Berhan 2014; Getahun et al. 2006). Category scores were then assigned 
to participants based on the sum of complications accumulated within each class of antenatal risk. The data were then reanalyzed using the same sociodemographic and medical variables as covariates; however, we substituted the four sums of risk scores for the single composite sum of nine antenatal complications.

\section{Results}

Table 3 shows analyses of performance for the total sample and for the subsample without significant brain injury cases.
As shown, the antenatal complications score was inversely related to the FSIQ, both before $(p=0.002)$ and after $(p=0.003)$ exclusion of brain injury cases. Analyses of preacademic performance revealed similar associations between antenatal risk and both CL and AP scores, before ( $p=0.001$ and $p=0.001$, respectively) and after ( $p=.002$ and $p=0.005$, respectively) excluding brain injury cases. No significant relationships were observed between cumulative antenatal risk and the VMI, yet the VMI was linked to gestational age $(p=0.042$ and 0.012). Added variable plots, depicting relationships between residualized antenatal risk scores and outcome

Table 3 Explanatory model: Antenatal risk and kindergarten readiness in full sample and after exclusion of significant brain injury cases

\begin{tabular}{|c|c|c|c|c|c|c|c|c|c|}
\hline & \multirow[t]{2}{*}{ Variables } & \multicolumn{4}{|c|}{ Total sample $(N=160)^{\mathrm{e}}$} & \multicolumn{4}{|c|}{$\begin{array}{l}\text { Subsample without Significant brain injury } \\
(N=149)^{\mathrm{f}}\end{array}$} \\
\hline & & $t$ & $d f$ & $p$ & $\Delta \mathrm{R}^{2(\mathrm{~g})}$ & $t$ & $d f$ & $p$ & $\Delta \mathrm{R}^{2(\mathrm{~g})}$ \\
\hline \multirow[t]{6}{*}{ Intellectual (Full Scale IQ) ${ }^{\mathrm{a}}$} & SES & 4.13 & 118.80 & $<.001$ & .122 & 4.65 & 115.92 & $<.001$ & .152 \\
\hline & $\operatorname{Sex}(F>M)$ & 2.26 & 131.35 & .024 & .036 & 3.13 & 134.22 & .002 & .077 \\
\hline & Gestational age & 1.29 & 139.79 & .199 & & 1.46 & 130.79 & .143 & \\
\hline & Antenatal complications ${ }^{\mathrm{h}, \mathrm{i}}$ & -3.15 & 124.67 & .002 & .068 & -2.98 & 109.72 & .003 & .067 \\
\hline & Perinatal complications ${ }^{\mathrm{j}}$ & 0.39 & 153.96 & .697 & & 1.35 & 145.99 & .176 & \\
\hline & IQ test edition & -2.19 & 109.54 & .029 & .037 & -1.29 & 102.96 & .196 & \\
\hline \multirow[t]{5}{*}{ Preliteracy (Core Language) ${ }^{\mathrm{b}}$} & SES & 3.74 & 124.88 & $<.001$ & .103 & 4.25 & 118.85 & $<.001$ & .142 \\
\hline & $\operatorname{Sex}(F>M)$ & 2.39 & 144.29 & .017 & .024 & 2.87 & 117.13 & .004 & .036 \\
\hline & Gestational age & 0.54 & 142.57 & .590 & & 0.77 & 136.30 & .445 & \\
\hline & Antenatal complications $\mathrm{s}^{\mathrm{h}, \mathrm{i}}$ & -3.36 & 122.84 & .001 & .085 & -3.11 & 129.20 & .002 & .069 \\
\hline & Perinatal complications ${ }^{\mathrm{j}}$ & -0.59 & 158.55 & .554 & & 0.06 & 144.32 & .949 & \\
\hline \multirow[t]{5}{*}{ Numeracy (Applied Problems) ${ }^{\mathrm{c}}$} & SES & 4.06 & 133.52 & $<.001$ & .108 & 4.48 & 119.51 & $<.001$ & .133 \\
\hline & $\operatorname{Sex}(F>M)$ & 3.44 & 151.43 & .001 & .073 & 3.47 & 147.57 & .001 & .082 \\
\hline & Gestational age & 0.38 & 129.00 & .707 & & 0.51 & 126.65 & .612 & \\
\hline & Antenatal complications ${ }^{\mathrm{h}, \mathrm{i}}$ & -3.28 & 121.90 & .001 & .071 & -2.80 & 93.39 & .005 & .059 \\
\hline & Perinatal complications ${ }^{\mathrm{j}}$ & -0.88 & 147.39 & .380 & & -0.45 & 147.77 & .653 & \\
\hline \multirow[t]{5}{*}{ Prewriting (Visual Motor Integration) ${ }^{\mathrm{d}}$} & SES & 3.25 & 131.99 & .001 & .075 & 3.69 & 122.46 & $<.001$ & .109 \\
\hline & $\operatorname{Sex}(F>M)$ & 3.92 & 159.41 & $<.001$ & .093 & 4.36 & 131.82 & $<.001$ & .097 \\
\hline & Gestational age & 2.04 & 143.40 & .042 & .012 & 2.52 & 131.04 & .012 & .038 \\
\hline & Antenatal complications $\mathrm{s}^{\mathrm{h}, \mathrm{i}}$ & -1.14 & 117.05 & .254 & & -0.40 & 125.57 & .690 & \\
\hline & Perinatal complications ${ }^{\mathrm{j}}$ & 0.42 & 159.28 & .674 & & 1.52 & 146.63 & .130 & \\
\hline
\end{tabular}

${ }^{\mathrm{a}}$ Wechsler Preschool and Primary Scale of Intelligence (III or IV). ${ }^{\mathrm{b}}$ Clinical Evaluation of Language Fundamentals - Preschool-2. ${ }^{\mathrm{c}}$ Woodcock Johnson III. ${ }^{d}$ Peabody Developmental Motor Scales-2. ${ }^{\text {e }}$ Three of 160 participants were unable to complete tasks required for scoring on any of the outcome measures. Of the remaining 157 cases, two failed to obtain a score on the FSIQ (one with brain injury), nine on CL ( 3 with brain injury), seventeen on AP (three with brain injury), six on the VMI. ${ }^{\mathrm{f}}$ Of the 148 "non-neurological" cases completing tasks required for a score on at least one of the four outcome measures, one $(.06 \%)$ could not obtain a score on the FSIQ, six (4.05\%) on CL, fourteen (9.45\%) on AP, six (4.05\%) on the VMI. ${ }^{\mathrm{g}}$ Computation of $\Delta \mathrm{R}^{2}$ based on Snijders and Bosker (1999, pp. 102-103). ${ }^{\mathrm{h}}$ Antenatal complications score is a composite of presence $(=1)$ vs. absence $(=0)$ of nine complications: placental abruption, placenta previa, chorioamnionitis, diabetes, hypertension in pregnancy, HELLP syndrome (hemolysis, elevated liver enzymes, low platelet count), hypothyroidism, preterm premature rupture of the membranes (PPROM) $>12 \mathrm{~h}$, and intrauterine growth restriction. ${ }^{i}$ Due to the paucity of cases with four antenatal complications, analyses were repeated with three and four complications combined into a single category ( $\geq 3$ ), with similar findings for the full sample: $t(131.72)=-2.67, p=.008 ; t(130.47)=-2.967, p=.003 ; t(109.00)=-2.881, p=.004 ; t(122.60)=1.537$, $p=.217$ for FSIQ, CL, AP, and VMI, respectively. For the "non-neurological" subsample: $t(118.12)=-2.745, p=.006 ; t(134.26)=-2.920, p=.004$; $t(104.00)=-2.580, p=.010 ; t(125.02)=-.419, p=.676$ for FSIQ, CL, AP, and VMI. ${ }^{j}$ Perinatal complications score is a composite of nine complications: anemia, bronchopulmonary dysplasia, bacterial infection, hyaline membrane disease, hyperbilirubinemia, hypoglycemia, intracranial pathology, patent ductus arteriosus, and supplemental oxygen requirement following discharge 
(FSIQ, CL, and AP) scores are shown in Online Resource 6 (a-c).

Supplemental analyses revealed that using an expanded model, with three additional predictors (multiple birth, school attendance, and bilingualism), did not meaningfully alter the nature of the relationships found between antenatal complications and either the FSIQ $(t[103.12]=-2.52, p=0.012), \mathrm{CL}$ $(t[127.74]=-2.92, p=0.004)$ AP $(t[85.17]=-2.53, p=$ $0.012)$, or the VMI $(t[112.60]=-0.05, p=0.963)$. Additionally, the use of birth weight SD (birth weight standardized for gestational age according to reference norms provided by Kramer et al. 2001) as a distinct predictor in our model, neither contributed $(\mathrm{t}[146.988]=-0.68, p=$ $0.49 ; \mathrm{t}[144.138]=0.21, p=0.83$; and $\mathrm{t}[131.93]=0.348$, $p=.73$ for FSIQ, CL, and AP) nor meaningfully altered $(\mathrm{t}$ $[104.487]=-2.71, p=.007 ; \mathrm{t}[121.74]=-2.94, p=0.003$; and $\mathrm{t}[92.28]=-2.67, p=0.008$ for FSIQ, CL, and AP) the relationships between antenatal complications and outcome measures.

Table 4 shows the relative contribution of each of the four categories of antenatal risk to the four performance measures; the proportion of variance explained $\left(\Delta R^{2}\right)$ is provided for statistical effects with $p<0.10$. As the table reveals, intraamniotic infection risk was significantly related to the FSIQ $(p=0.016), \mathrm{CL}(p=0.043)$, and AP $(p=0.012)$, whereas conditions associated with placental insufficiency were significantly related to CL $(p=0.047)$, with trends for associations with the FSIQ and AP ( $p=0.071$ and 0.064 , respectively). Maternal endocrine dysfunction was associated with CL $(p=0.040)$, and disorders associated with uteroplacental bleeding were significantly related to the FSIQ $(p=0.048)$.

\section{Discussion}

Following statistical adjustment for sociodemographic and perinatal confounds, the sum of nine, relatively common, antenatal complications remained a significant source of variability in preterm-born preschoolers' cognitive and academic performance. Exploration of the relative outcome contribution of four classes of antenatal risk revealed that complications associated with intra-amniotic infection, placental insufficiency and uteroplacental bleeding accounted for $4.8 \%, 2.3 \%$ and $3.4 \%$ of IQ variance, respectively, altogether $10.5 \%$ of variability in kindergarten cognitive readiness. Similarly, complications associated with maternal endocrine dysfunction, intraamniotic infection, and placental insufficiency accounted for $3.8 \%, 3.3 \%$ and $2.7 \%$, of the variance in language skills, respectively, altogether $9.8 \%$ of variability in literacy readiness. Complications associated with intra-amniotic infection risk and placental insufficiency contributed $5.9 \%$ and $2.5 \%$ of the variance in early number concepts, respectively, a combined share of $8.4 \%$ to variability in numeracy readiness.
Hence, when considered separately, each of the four risk categories accounts for a small slice of outcome variance in one or more preacademic domains (except visual-motor integration). Yet in aggregate they accounted for 8.4-10.5\% of the variance in kindergarten readiness of preschoolers free of major handicaps, consistent with effect sizes of moderate magnitude (Cohen 1992).

Interestingly, amongst the four categories of antenatal risk examined here, intra-amniotic infection was the most consistent contributor to kindergarten readiness. These findings are compatible with recent evidence that inflammation, including chorioamnionitis, contributes to preterm CNS injury and is also an independent risk factor for brain injury in the term infant (Yellowhair et al. 2018). These results are also consistent with reports of marked increase in the probability of unexplained cerebral palsy in the presence of antenatal inflammation-infection (Horvath et al. 2012). In this context, one should note that a sizeable proportion of the mothers in our sample received antibiotics prophylaxis for prevention of early onset neonatal infection (see Online Resource 2). In a recent integrative review of the literature, Braye et al. (2018) highlighted the observed decrease in incidence of early onset infection since the introduction of intrapartum antibiotic prophylaxis, as well as findings of randomized controlled studies documenting effectiveness. At the same time, however, Braye and colleagues emphasized that the longer-term health implications of prophylaxis for early onset infection are unknown. It is difficult, therefore, if not impossible, to draw conclusions regarding the potential implications of antibiotic prophylaxis on kindergarten readiness until such data become available. Nonetheless, our findings revealed that each of the four classes of antenatal risk studied here contributed significantly to explaining performance variance on one or more kindergarten readiness domains. The putative influence of each antenatal risk category on brain maturation trajectory and cognitivebehavioral development may be conceptualized within the broad framework of fetal programming of disease (Buss et al. 2012; Zeltser and Leibel 2011; Myatt 2006; Andersen et al. 2014; Miller et al. 2016; Godfrey 2002). However, the biological mechanisms mediating the relationship between cumulative antenatal risk and kindergarten readiness require specification. Antenatal stressors lead to placental adaptive responses to the variations in the maternal-fetal environment (Myatt 2006). These responses, in turn, are followed by fetal adaptations expressed via vascular, metabolic or endocrine changes that permanently modify bodily structure or function (Hocher et al. 2001). The precise nature and sequence of the biological changes mediating deficits in cognitive-behavioral functioning have yet to be elucidated. To accomplish this goal, future investigations should incorporate measures of potential sequential mediators, including indexes of placental size or function, intrauterine cerebral development, and postnatal brain structure or function. 
Table 4 Explanatory model: Classes of antenatal risk and kindergarten readiness in $149^{\mathrm{a}}$ cases without significant brain injury

\begin{tabular}{|c|c|c|c|c|c|c|c|c|}
\hline \multirow[b]{2}{*}{ Predictors } & \multicolumn{4}{|c|}{ Intellectual (Full Scale IQ) ${ }^{\mathrm{b}}$} & \multicolumn{4}{|c|}{ Numeracy (Applied Problems) ${ }^{\mathrm{d}}$} \\
\hline & $t$ & $d f$ & $p$ & $\Delta \mathrm{R}^{2}$ & $t$ & $d f$ & $p$ & $\Delta \mathrm{R}^{2}$ \\
\hline SES & 4.49 & 114.50 & $<.001$ & .144 & 4.21 & 116.96 & $<.001$ & .118 \\
\hline $\operatorname{Sex}(F>M)$ & 3.00 & 133.10 & .003 & .070 & 3.49 & 145.62 & .001 & .084 \\
\hline Gestational age & 1.28 & 128.61 & .201 & & 0.26 & 122.88 & .795 & \\
\hline Perinatal complications ${ }^{\mathrm{f}}$ & 1.28 & 146.19 & .200 & & -0.50 & 146.23 & .620 & \\
\hline Intra-amniotic infection risk ${ }^{\mathrm{g}}$ & -2.40 & 120.32 & .016 & .048 & -2.56 & 119.12 & .012 & .059 \\
\hline Placental insufficiency ${ }^{\text {ih }}$ & -1.81 & 123.14 & .071 & .023 & -1.85 & 97.66 & .064 & .025 \\
\hline Maternal endocrine dysfunction ${ }^{\mathrm{i}}$ & -1.19 & 87.31 & .236 & & -1.47 & 83.75 & .141 & \\
\hline Uteroplacental bleeding risk ${ }^{\mathrm{j}}$ & -1.98 & 94.30 & .048 & .034 & -0.75 & 85.89 & .455 & \\
\hline \multirow[t]{2}{*}{ IQ test edition } & -1.36 & 101.84 & .175 & & & & & \\
\hline & \multicolumn{4}{|c|}{ Preliteracy (Core Language) ${ }^{c}$} & \multicolumn{4}{|c|}{ Prewriting (Visual Motor Integration) ${ }^{\mathrm{e}}$} \\
\hline Predictors & $t$ & $d f$ & $p$ & $\Delta \mathrm{R}^{2}$ & $t$ & $d f$ & $p$ & $\Delta \mathrm{R}^{2}$ \\
\hline SES & 4.23 & 118.63 & $<.001$ & .138 & 3.48 & 118.21 & .001 & .097 \\
\hline $\operatorname{Sex}(F>M)$ & 2.71 & 113.75 & .007 & .030 & 4.40 & 131.02 & $<.001$ & .103 \\
\hline Gestational age & 0.74 & 135.92 & .460 & & 2.38 & 132.62 & .018 & .032 \\
\hline Perinatal complications ${ }^{\mathrm{f}}$ & -0.05 & 144.22 & .964 & & 1.50 & 148.30 & .135 & \\
\hline Intra-amniotic infection risk ${ }^{\mathrm{g}}$ & -2.02 & 129.16 & .043 & .033 & -0.79 & 125.60 & .430 & \\
\hline Placental insufficiency ${ }^{\text {h }}$ & -2.00 & 141.95 & .047 & .027 & -0.72 & 130.43 & .475 & \\
\hline Maternal endocrine dysfunction ${ }^{\mathrm{i}}$ & -2.05 & 103.89 & .040 & .038 & 1.07 & 95.70 & .283 & \\
\hline Uteroplacental bleeding risk ${ }^{\mathrm{j}}$ & -1.28 & 108.90 & .200 & & -0.58 & 101.60 & .560 & \\
\hline
\end{tabular}

${ }^{a}$ Of the 148 "non-neurological" cases who completed task(s) required for a score on at least one of the four outcome measures, one (.06\%) could not obtain a score on the Full Scale IQ (FSIQ), six (4.05\%) on Core Language (CL), fourteen (9.45\%) on Applied Problems (AP), and six (4.05\%) on Visual Motor Integration (VMI). Multiple imputation was applied to replace missing FSIQ, CL, AP, and VMI scores. ${ }^{\text {b }}$ Wechsler Preschool and Primary Scale of Intelligence - III or IV. ${ }^{\mathrm{c}}$ Clinical Evaluation of Language Fundamentals - Preschool II. ${ }^{\mathrm{d}}$ Woodcock Johnson III. ${ }^{\mathrm{e}}$ Peabody Developmental Motor Scales-2. ${ }^{f}$ Perinatal complications score is a composite score reflecting presence $(=1)$ vs. absence $(=0)$ of nine complications: anemia, bronchopulmonary dysplasia, bacterial infection, hyaline membrane disease, hyperbilirubinemia, hypoglycemia, intracranial pathology, patent ductus arteriosus, and supplemental oxygen requirement following discharge. ${ }^{\mathrm{g}}$ Intra-amniotic infection risk score is a composite of two complications believed to share etiological pathways: preterm premature rupture of membranes (PPROM) $>12 \mathrm{~h}$ and histological chorioamnionitis. ${ }^{\mathrm{h}}$ Placental insufficiency score is a composite of three complications thought to share etiological pathways: maternal hypertension, HELLP syndrome (hemolysis, elevated liver enzymes, low platelet count), and intrauterine growth restriction. ${ }^{\mathrm{i}}$ Maternal endocrine dysfunction score is a composite of two complications found to share some etiological pathways: maternal diabetes and hypothyroidism. ${ }^{j}$ Uteroplacental bleeding risk score is a composite of two complications sharing antenatal symptomatology: placental abruption and placenta previa

Our findings are consistent with Zeltser and Leibel's (2011) observations that dissimilar intrauterine stress factors may nonetheless lead to similar fetal outcomes because they activate related mechanisms of placental adaptation (Fowden et al. 2009) which, in turn, shape the trajectory of fetal brain development (Buss et al. 2012). The thesis that diverse insults converge on similar unfavorable outcomes (a relationship mediated by fetal brain programming) is compatible with the notion that the amalgamation of antenatal complications into a single index or several classes of risk may offer an important tool for the study of individual differences among pretermborn children in the severity of neurocognitive deficits.

Antenatal complications contributed less than SES to explaining outcome variance, although effect magnitude was typically moderate for both variables. Girls outperformed boys on all measures, consistent with other reports of female outcome advantage following preterm birth (Lauterbach et al.
2001). Similar to Foster-Cohen et al. (2010), we did not find significant associations between the sum of perinatal complications and kindergarten readiness in this young age group. However, gestational age, often considered a proxy for perinatal risk, was found to be linked to development of visualmotor integration skills even after we excluded cases with evidence of significant brain injury from the analyses. Additionally, there was a weak trend $(p<0.15$; Table 3$)$ for a relationship between gestational age and global intellectual skills. The absence of the oft-reported significant relationships between the degree of prematurity and the remaining outcome measures (e.g., Heuvelman et al. 2018; a recent epidemiological investigation) was somewhat unexpected. It is possible that within a restricted gestational age range, where values $\geq 34$ weeks were truncated, the relationships between gestational age and preschool performance are more difficult to demonstrate whereas the adverse influence of other risk 
factors (e.g., antenatal complications) becomes more apparent. Interestingly, the absence of gestational age effects on several performance measures employed here seems consistent with findings from a recent meta-analysis of the long-term cognitive and academic performance of children born with various degrees of prematurity (Allotey et al. 2018). The quantitative integration revealed a miniscule, if any, effect of lower gestational age. Children born between 28 and 34 weeks performed almost as poorly as those born $<28$ weeks.

As noted earlier, our findings of an association between cumulative antenatal risk and kindergarten readiness are consistent with the fetal programming hypothesis. Researchers of fetal programming have typically studied the role of the prenatal environment without taking into consideration the perinatal, or postnatal environment (Grant et al. 2015). In the current study, however, we statistically adjusted cumulative antenatal risk for perinatal complications and for socioeconomic status in effort to account for confounding influences occurring after birth.

Limitations of this study include the retrospective nature of the participant identification and recruitment component. Medical data were also collected retrospectively, yet interrater reliability for information obtained from medical records was excellent. The difficulty of a small number of participants to complete various tasks is not surprising, given the combination of young age and increased biomedical risk in our sample. The percentage of missing outcome data for the 148 children without significant neurological injury completing $\geq$ one task ranged from negligible $(0.06 \%)$ for cognitive readiness (FSIQ) to moderate $(9.45 \%)$ for numerical readiness (AP). The amount of missing data for three of the four outcome measures was nonetheless small $(<5.0 \%)$. Although our sample included children with a wide range of socioeconomic circumstances, the mean SES and educational characteristics of the sample revealed a greater representation of middle-class strata, thereby reducing the potentially confounding effects of socioeconomic adversity on kindergarten readiness. Nonetheless, because to some extent generalizability to lower strata was traded-off for improved internal validity, our findings may have underestimated statistical effects in samples with greater representation of the lower end of the socioeconomic scale. The cross-sectional nature of this investigation precluded examination of the generalizability of the findings to elementary school readiness and beyond.

In the current investigation antenatal risk accounted for up to $10.5 \%$ of the variance in kindergarten readiness. Antenatal risk estimation was based on a simple frequency count of common complications. A more sensitive measure of risk may be developed to take the severity of discrete complications within each of the four antenatal risk categories examined here into account. Increased sensitivity, in turn, may serve to enhance the magnitude of the statistical effects observed here between antenatal risk and emergent academic skills. As noted above, we statistically adjusted for degree of gestational maturity and for the presence of nine common perinatal risk factors (Table 2) in order to estimate the unique portion of outcome variance that is attributable to cumulative antenatal risk. We further examined the data with and without 'neurological' cases, based on both ultrasound evidence obtained in the NICU and subsequent evidence of cerebral palsy. Nonetheless, one may argue that the full effect of confounding perinatal risk factors such as chronic lung disease or germinal matrix hemorrhage may become evident in the longer term only, when academic performance demands are increased. A longitudinal study of educational attainment is best suited to address this issue.

The significance of early academic skills as the foundation of scholastic achievement has been established in multiple follow-up investigations of typical kindergartners to the early school years (e.g., Pagani et al. 2010; Romano et al. 2010; Grissmer et al. 2010). Moreover, developmental continuity has been demonstrated between preschool quantitative and oral-language skills and elementary school attainment in math and reading, in a variety of student populations (e.g., Davison et al. 2011; Manfra et al. 2014; Manfra et al. 2017; Nguyen et al. 2016). The existence of robust developmental continuity between pre-academic and academic skill levels supports the notion that the same factors that explain variability in emergent academic skills also account for variability in mastery of both the reading process and basic arithmetic in the early elementary-school years. Based on our findings, it is therefore likely that differences in scholastic achievement among graduates of the NICU are partly explained by cumulative antenatal risk. A follow-up study of preterm-born preschoolers, extending to early school-age and beyond, will be needed to support this thesis.

Acknowledgements The authors thank Beth Kring and Tammy Swails for their help in data collection. Evaluations and testing materials were funded in part by the Merrill-Palmer Skillman Institute. None of the authors has a known conflict of interest concerning this manuscript.

Funding This work was supported in part by funding from the Merrill Palmer Skillman Institute, Wayne State University, 71 East Ferry, Detroit, MI 48202.

\section{Compliance with Ethical Standards}

Conflict of Interest The authors declare that they have no conflicts of interest.

\section{References}

Aarnoudse-Moens, C. S. H., Oosterlaan, J., Duivenvoorden, H. J., van Goudoever, J. B., \& Weisglas-Kuperus, N. (2011). Development of preschool and academic skills in children born very preterm. The Journal of Pediatrics, 158(1), 51-56. https://doi.org/10.1016/j. jpeds.2010.06.052. 
Alfonso, V. C., \& Flanagan, D. P. (2002). Comparative features of comprehensive achievement batteries, Woodcock-Johnson III: Assessment service bulletin $N^{\circ} 5$. Itasca: Riverside.

Allotey, J., Zamora, J., Cheong-See, F., Kalidini, M., Arroyo-Manzano, D., Esztalos, E., et al. (2018). Cognitive, motor, behavioural and academic performances of children born preterm: A meta-analysis and systematic review involving 64,061 children. British Journal of Obstetrics and Gynaecology, 125(1), 16-25. https://doi.org/10. 1111/1471-0528.14832.

Andersen, S. L., Olsen, J., Wu, C. S., \& Laurberg, P. (2014). Psychiatric disease in late adolescence and young adulthood. Foetal programming by maternal hypothyroidism? Clinical Endocrinology, 81(1), 126-133. https://doi.org/10.1111/cen.12415.

Andersen, S. L., Andersen, S., Vestergaard, P., \& Olsen, J. (2018). Maternal thyroid function in early pregnancy and child neurodevelopmental disorders: A Danish nationwide case-cohort study. Thyroid, 28(4), 537-346. https://doi.org/10.1089/thy.2017. 0425.

Barker, D. J. P. (1998). In utero programming of chronic disease. Clinical Sciences, 95(2), 115-128.

Barnhart, L. (2015). HELLP syndrome and the effects on the neonate. Neonatal Network, 34(5), 269-273. https://doi.org/10.1891/07300832.34.5.269.

Baron, I. S., Erickson, K., Ahronovich, M. D., Coulehan, K., Baker, R., \& Litman, F. R. (2009). Visuospatial and verbal fluency relative deficits in "complicated" late-preterm preschool children. Early Human Development, 85(12), 751-754. https://doi.org/10.1016/j. earlhumdev.2009.10.002.

Baron, I. S., Erickson, K., Ahronovich, M. D., Baker, R., \& Litman, F. R. (2011). Cognitive deficit in preschoolers born late-preterm. Early Human Development, 87(2), 115-119. https://doi.org/10.1016/j. earlhumdev.2010.11.010

Batton, D. G., DeWitte, D. B., \& Pryce, C. J. (2011). One hundred consecutive infants born at 23 weeks and resuscitated. American Journal of Perinatology, 28(4), 299-304. https://doi.org/10.1055/s0030-1268714

Berhan, Y. (2014). Predictors of perinatal mortality associated with placenta previa and placental abruption: An experience from a low income country. Journal of Pregnancy, 2014, 307043. https://doi. org/10.1155/2014/307043.

Braye, K., Ferguson, J., Davis, D., Catling, C., Monk, A., \& Foureur, M. (2018). Effectiveness of intrapartum antibiotic prophylaxis for earlyonset group B streptococcal infection: An integrative review. Women and Birth, 31(4), 244-253. https://doi.org/10.1016/j. wombi.2017.10.012.

Buss, C., Entringer, S., \& Wadhwa, P. D. (2012). Fetal programming of brain development: Intrauterine stress and susceptibility to psychopathology. Science Signaling, 5 (245, pt7). https://doi.org/10.1126/ scisignal.2003406.

Caravale, B., Tozzi, C., Albino, G., \& Vicari, S. (2005). Cognitive development in low risk preterm infants at 3-4 years of life. Archives of Disease in Childhood Fetal and Neonatal Edition, 90, F474-F479. https://doi.org/10.1136/adc.2004.070284.

Carmody, D. P., Bendersky, M., Dunn, S. M., DeMarco, J. K., Hegyi, T., Hiatt, M., \& Lewis, M. (2006). Early risk, attention, and brain activation in adolescents born preterm. Child Development, 77(2), 384 394. https://doi.org/10.1111/j.1467-8624.2006.00877.x.

Cohen, J. (1992). A power primer. Psychological Bulletin, 112(1), 155159. https://doi.org/10.1037/0033-2909.112.1.155.

Davis, E. P., \& Sandman, C. A. (2012). Prenatal psychobiological predictors of anxiety risk in preadolescent children. Psychoneuroendocrinology, 37(8), 1224-1233. https://doi.org/10. 1016/j.psyneuen.2011.12.016.

Davison, M. D., Hammer, C., \& Lawrence, F. R. (2011). Associations between preschool language and first grade reading outcomes in bilingual children. Journal of Communication Disorders, 44(4), 444-458. https://doi.org/10.1016/j.jcomdis.2011.02.003.

Downes, K. L., Sheneassa, E. D., \& Grantz, K. L. (2017). Neonatal outcomes associated with placental abruption. American Journal of Epidemiology, 186(12), 1319-1328. https://doi.org/10.1093/aje/ kwx202.

Eide, M. G., Øyen, N., Skjaerven, R., Irgens, L. M., Bjerkedal, T., \& Nilsen, S. T. (2005). Breech delivery and intelligence: A population-based study of 8,738 breech infants. Obstetrics and Gynecology, 105(1), 4-11. https://doi.org/10.1097/01.AOG. 0000149743.80837.d3.

Folio, M. R., \& Fewell, R. R. (2000). Peabody developmental motor scales-2nd edition. PRO-ED: Austin, TX.

Foster-Cohen, S. H., Friesen, M. D., Champion, P. R., \& Woodward, L. J. (2010). High prevalence/low severity language delay in preschool children born very preterm (2010). Journal of Developmental and Behavioral Pediatrics, 31(8), 658-667. https://doi.org/10.1097/ DBP.0b013e3181e5ab7e.

Fowden, A. L., Sferruzzi-Perri, A. N., Coan, P. M., Constancia, M., \& Burton, G. J. (2009). Placental efficiency and adaptation: Endocrine regulation. The Journal of Physiology, 587(pt.14), 3459-3472. https://doi.org/10.1113/jphysiol.2009.173013.

Fraser, A., Nelson, S. M., Macdonald-Wallis, C., \& Lawlor, D. A. (2012). Associations of existing diabetes, gestational diabetes, and glycosuria with offspring IQ and educational attainment: The Avon longitudinal study of parents and children. Experimental Diabetes Research, 963735. https://doi.org/10.1155/2012/963735.

Getahun, D., Oyelese, Y., Salihu, H. M., \& Ananth, C. V. (2006). Previoius cesarean delivery and risks of placenta previa and placental abruption. Obstetrics \& Gynecology, 107(4), 771-778. https:// doi.org/10.1097/01.AOG.0000206182.63788.80.

Godfrey, K. M. (2002). The role of the placenta in fetal programming-a review. Placenta, Suppl A, S20-S27. https://doi.org/10.1053/plac. 2002.0773 .

Grant, K.-A., Sandman, C. A., Wing, D. A., Dmitrieva, J., \& Davis, E. P. (2015). Prenatal programming of postnatal susceptibility to memory impairments: A developmental double jeopardy. Psychological Science, 26(7), 1054-1062. https://doi.org/10.1177/ 0956797615580299

Grissmer, D., Grimm, K. J., Aiyer, S. M., Murrah, W. M., \& Steele, J. S. (2010). Fine motor skills and early comprehension of the world: Two new readiness indicators. Developmental Psychology, 46(5), 1008-1017. https://doi.org/10.1037/a0020104.

Haddow, J. E., Craig, W. Y., Neveux, L. M., Palomaki, G. E., LambertMesserlian, G., Malone, F. D., \& D'Alton, M. E. (2016). Free thyroxine during early pregnancy and risk for gestational diabetes. PLoS One, 11(2), e0149065. https://doi.org/10.1371/journal.pone. 0149065.

Heuvelman, H., Abel, K., Wicks, S., Gardner, R., Johnstone, E., Lee, B., Magnusson, C., Dalman, C., \& Rai, D. (2018). European Journal of Epidemiology, 33, 667-678. https://doi.org/10.1007/s10654-0170340-1.

Hocher, B., Slowinski, T., Bauer, C., \& Halle, H. (2001). Nephrology Dialysis Transplantation, 16(6), 1298-1299. https://doi.org/10. 1093/ndt/16.6.1298.

Hollingshead, A. B. (1975). Four-factor index of social status. New Haven: Yale University.

Horvath, B., Grasselly, M., Bodecs, T., Boncz, I., \& Bodis, J. (2012). European Journal of Obstetrics \& Gynecology and Reproductive Biology, 163(2), 160-164. https://doi.org/10.1016/j.ejogrb.2012. 05.012 .

Huang, J., Zhu, T., Qu, Y., \& Mu, D. (2016). Prenatal, perinatal and neonatal risk factors for intellectual disability: A systemic review and meta-analysis. PLoS One, 11(4), e0153655. https://doi.org/10. 1371/journal.pone.0153655. 
Ismail, M. A., Nagib, N., Ismail, T., \& Cibils, L. A. (1999). Comparison of vaginal and cesarean section delivery for fetuses in breech presentation. Journal of Perinatal Medicine, 27(5), 339-351. https:// doi.org/10.1515/JPM.1999.047.

Kerr-Wilson, C. O., Mackay, D. F., Smith, G. C. S., \& Pell, J. P. (2012). Meta-analysis of the association between preterm delivery and intelligence. Journal of Public Health, 34(2), 209-216. https://doi.org/ 10.1093/pubmed/fdr024.

Kramer, M. S., Platt, R. W., Wen, S. W., Joseph, K. S., Allen, A., Abrahamowicz, M., et al. (2001). A new and improved populationbased Canadian reference for birth weight for gestational age. Pediatrics, 108(2), E35. https://doi.org/10.1542/peds.108.2.e35.

Lauterbach, M. D., Raz, S., \& Sander, C. J. (2001). Neonatal hypoxic risk in preterm birth infants: The influence of sex and severity of respiratory distress on cognitive recovery. Neuropsychology, 15(3), 411420. https://psycnet.apa.org/doi/10.1037/0894-4105.15.3.411.

Liu, Y., Chen, H., Jing, C., \& Li, F. (2018). The association between maternal subclinical hypothyroidism and growth, development, and childhood intelligence: A meta-analysis. Journal of Clinical Research in Pediatric Endocrinology, 10(2), 153-161. https://doi. org/10.4274/jcrpe.4931.

Maher, G. M., O’Keeffe, G. W., Kennuy, L., Kearney, P. M., Dinan, T. G., \& Khashan, A. (2017). Hypertensive disorders of pregnancy and risk of neurodevelopmental disorders - A systematic review and meta-analysis protocol. BMJ Open, 7(10), e018313. https://doi.org/ 10.1136/bmjopen-2017-018313.

Manfra, L., Dinehart, L. H. B., \& Sembiante, S. F. (2014). Associations between counting ability in preschool and mathematic performance in first grade among a sample of ethnically diverse, low-income children. Journal of Research in Childhood Education, 28, 101114. https://doi.org/10.1080/02568543.2013.850129.

Manfra, L., Squires, C., Dinehart, L. H. B., Bleiker, C., Hartman, S. C., \& Winsler, A. (2017). Preschool writing and premathematics predict grade 3 achievement for low-income, ethnically diverse children. The Journal of Educational Research, 110(5), 528-537. https:// doi.org/10.1080/00220671.2016.1145095.

Miller, S. L., Huppi, P. S., \& Mallard, C. (2016). The consequences of fetal growth restriction on brain structure and neurodevelopmental outcome. The Journal of Physiology, 594(4), 807-823. https://doi. org/10.1113/JP271402.

Morsing, E., Asard, M., Ley, D., Stjernqvist, K., \& Marsal, K. (2011). Cognitive function after intrauterine growth restriction and very preterm birth. Pediatrics, 127(4), e874-e872. https://doi.org/10.1542/ peds.2010-1821.

Myatt, L. (2006). Placental adaptive responses and fetal programming. The Journal of Physiology, 572(pt 1), 25-30. https://doi.org/10. 1113/jphysiol.2006.104968.

Nguyen, T., Watts, T. W., Duncan, G. J., Clements, D. H., Sarama, J. S., Wolfe, C., \& Spitler, M. E. (2016). Which preschool mathematics competencies are most predictive of fifth grade achievement? Early Child Research Quarterly, 36, 550-560. https://doi.org/10.1016/j. ecresq.2016.02.003.

Pagani, L. S., Fitzpatrick, C., Archambault, I., \& Janosz, M. (2010). School readiness and later achievement: A French Canadian replication and extension. Developmental Psychology, 46(5), 984-994. https://doi.org/10.1037/a0018881.

Pinto, S., Malheiro, M. F., Vaz, A., Rodrigues, T., Montenegro, N., \& Guimaraes, H. (2018). Neonatal outcome in preterm deliveries before 34-week gestation - The influence of the mechanism of labor onset (2018). Journal of Maternal-Fetal \& Neonatal Medicine, 21, 1-7. https://doi.org/10.1080/14767058.2018.1481038.

Romano, E., Babchishin, L., Pagani, L. S., \& Kohen, D. (2010). School readiness and later achievement: Replication and extension using a nationwide Canadian survey. Developmental Psychology, 46(5), 995-1007. https://doi.org/10.1037/a0018880.
Shalev, I., Caspi, A., Ambler, A., Belsky, D. W., Chapple, S., Cohen, H. J., et al. (2014). Perinatal complications and aging indicators by midlife. Pediatrics, 134, e1315-e1323. https://doi.org/10.1542/peds.2014-1669.

Shrout, P. E., \& Fleiss, J. L. (1979). Intraclass correlations: Uses in assessing rater reliability. Psychological Bulletin, 86(2), 420-428.

Snijders, T. A. B., \& Bosker, R. J. (1999). Multilevel Analysis: An introduction to basic and advanced multilevel modeling (pp. 102-103). London: Sage.

Stepan, H., Leo, H., Purz, S., Hockel, M., \& Horn, L.-C. (2005). Placental localization and expression of the cell death factors BNip3 and nix in preeclampsia, intrauterine growth retardation and HELLP syndrome. European Journal of Obstetrics \& Gynecology and Reproductive Biology, 122(2), 172-176. https://doi.org/10.1016/j.ejogrb.2005.01.017.

Stock, O., Gordon, L., Kapoor, J., Walker, S., Whitehead, C., Kaitu'uLino, T. J., et al. (2015). Chorioamnionitis occurring in women with preterm rupture of the fetal membranes is associated with a dynamic increase in mRNAs coding cytokines in the maternal circulation. Reproductive Science, 22(7), 852-859. https://doi.org/10.1177/ 1933719114565034.

Torrioli, M. G., Frisone, M. F., Bonvini, L., Luciano, R., Pasca, M. G., Lepori, R., et al. (2000). Perceptual-motor, visual and cognitive ability in very low birthweight preschool children without neonatal ultrasound abnormalities. Brain \& Development, 22(3), 163168. https://doi.org/10.1016/S0387-7604(00)00098-X.

Volpe, J. J. (2001). Intracranial-hemorrhage: Germinal matrixintraventricular hemorrhage of the premature infant. In J. J. Volpe (Ed.). Neurology of the newborn (4th ed., pp. 428-493). Philadelphia: W. B. Saunders.

Wade, M., Madigan, S., Akbari, E., \& Jenkins, J. M. (2015). Cumulative biomedical risk and social cognition in the second year of life: Prediction and moderation by responsive parenting. Frontiers in Psychology, 6(354). https://doi.org/10.3389/fpsyg.2015.00354.

Wechsler, D. (2002). Wechsler primary and preschool scale of IntelligenceTM (third edition WPPSITM-III). San Antonio: Harcourt Assessment.

Wechsler, D., Coalson, D., \& Raiford, S. (2012). Wechsler preschool and primary scale of intelligence (Fourth ed.). United States of America: The Psychological Corporation.

Weiner, E., Miremberg, H., Grinstein, E., Mizrachi, Y., Schreiber, L., Bar, J., \& Kovo, M. (2016). The effect of placenta previa on fetal growth and pregnancy outcome, in correlation with placental pathology. Journal of Perinatology, 36(12), 1073-1078. https://doi.org/10. 1038/jp.2016.140.

Whitehouse, A. J. O., Shelton, W. M. R., Ing, C., \& Newnham, J. P. (2014). Prenatal, perinatal, and neonatal risk factors for specific language impairment: A prospective pregnancy cohort study. Journal of Speech, Language, and Hearing Research, 57(4), 1418-1427. https://doi.org/10.1044/2014_JSLHR-L-13-0186.

Wiig, E., Secord, W., \& Semel, E. (2004). Clinical evaluation of language fundamentals: Preschool-2. San Antonio: Psychological Corporation.

Woodcock, R. W., McGrew, K. S., \& Mather, N. (2001). WoodcockJohnson tests of achievement. Itasca: Riverside Publishing.

Yellowhair, T. R., Noor, S., Maxwell, J. R., Anstine, C. V., Oppong, A. Y., Robinson, S., et al. (2018). Preclinical chorioamnionitis dysregulates CXCL1/CXCR2 signaling throughout the placentalfetal-brain axis. Experimental Neurology, 301(Pt B), 110-119. https://doi.org/10.1016/j.expneurol.2017.11.002 .

Zeltser, L. M., \& Leibel, R. L. (2011). Roles of the placenta in fetal brain development. Proceedings of the National Academy of Science, USA, 108(38), 15667-15668. https://doi.org/10.1073/pnas.1112239108.

Publisher's Note Springer Nature remains neutral with regard to jurisdictional claims in published maps and institutional affiliations. 